\title{
Effects of Display Configurations on Document Triage
}

\author{
Soonil Bae, Rajiv Badi, Konstantinos Meintanis, J. Michael Moore, Anna Zacchi, \\ Haowei Hsieh, Catherine C. Marshall*, and Frank M. Shipman \\ Center for the Study of Digital Libraries \& Department of Computer Science, \\ Texas A\&M University \\ shipmanecs . tamu . edu
}

\begin{abstract}
Document triage is the practice of quickly determining the merit and disposition of relevant documents. This practice involves selection of documents from a document overview and quick forms of reading: skimming, reading short portions of a longer document, and navigating through headings, indices, and tables of contents. Earlier studies of document triage practice showed considerable overhead related to window management during transitions between the document overview and reading interfaces. This study examines the impact of multiple display configurations on document triage practice. In particular, it compares (1) configurations with same and different size displays, and (2) configurations with and without user control over which activity is performed on which display. Results show a significant increase in the number of transitions between activities when a multi-display configuration is introduced although there is no significant difference between the different multiple display configurations. Additionally, user activity with a document was positively correlated with an overall assessment of document value.
\end{abstract}

\section{Introduction}

With the ubiquity of digital documents, users deal with multiple documents when they are looking up information on a particular topic. A student doing a literature survey typically uses a search engine to locate potentially relevant papers for the area of interest. She then skims, scans and evaluates the different documents, making comparisons and/or saving the references. She relies on what Joyce refers to as "successive attendings" to the same materials [5], rather than on scholarly reading and notetaking. Prior research on this triage activity suggests that during information triage, attentional resources are devoted to evaluating materials and organizing them, so they can be read and reread as they return to mind [7].

Document triage is the practice of quickly determining the merit and disposition of relevant documents - including web pages, periodical articles, and other published materials - that one may locate using a search engine, receive from an automated delivery mechanism, or obtain from a human intermediary. This practice necessarily involves quick forms of reading: skimming, reading short portions of a longer document, and navigating through structural elements such as headings, indices, or tables of contents. It also involves comparing documents, integrating results across

Microsoft Corporation, Redmond, Washington. 
documents, noticing missing information, and reconciling conflicting information. Although this kind of triage is related to activities such as managing email, or getting a quick answer to a question by finding the "right" document, there is an important distinction: document triage usually involves a current focal document (or documents) and a periphery or background of other documents relevant to the task at hand.

Document triage gives us a way to investigate tasks in which reading and attention shift from document to document to contextual overview. In other words, we are looking beyond intensive reading (engagement with a single document) to extensive reading (engagement with multiple documents at once) and to hyper-extensive reading (engagement with subdocument components and fragmentary information).

The central issues being investigated in this study are:

- How can display real-estate and multiple displays and devices best be used to facilitate this kind of reading and gathering task?

- How can productivity be defined and promoted in multiple document tasks in which readers must manage their attentional resources?

\section{Approach}

Our main motivation for this research stems from the results from the study detailed in [12]. This study characterized the shifting attention problem by using a standard document triage task - going through search results and selecting and organizing the items considered valuable. A notable aspect of the data is how many transitions, i.e. shifting attention between the overview (in this case the Visual Knowledge Builder [11]) and the full reading window (in this case, Internet Explorer), this task requires.

Table 1. Transitions between document overview and reading application in prior study

\begin{tabular}{|l|c|c|c|c|c|c|c|c|}
\hline Subject ID & 1 & 2 & 3 & 4 & 5 & 6 & 7 & 8 \\
\hline $\begin{array}{l}\text { Minutes spent on } \\
\text { triage activity }\end{array}$ & 64.1 & 54.2 & 22.0 & 22.8 & 93.5 & 80.2 & 63.8 & 61.7 \\
\hline $\begin{array}{l}\text { Transitions between } \\
\text { overview \& reading }\end{array}$ & 134 & 28 & 78 & 81 & 98 & 106 & 87 & 90 \\
\hline Transitions/minute & 2.1 & 0.5 & 3.5 & 3.6 & 1.0 & 1.3 & 1.4 & 1.5 \\
\hline
\end{tabular}

The data suggests that the number of transitions between the overview and reading applications is a profound source of interruption, especially since users had to rearrange or reorder windows at almost every transition. There is an average of more than one transition per minute for most users and this table does not include withinapplication navigation and reading-related navigation and manipulation that may be disruptive. It is instructive to examine several of the sessions individually to get a sense for this disruption. Subject 2 shows relatively few transitions in a 54 minute session; he or she sacrificed reading in favor of working on the triage task directly from the metadata provided in the overview. By contrast, Subject 4 shows the most transitions of any of the participants in the study. Subject 4 shifted between creating structure in the overview and reading Web pages to see what they were about. 
Several questions thus arise from looking at this data:

- Do people try to minimize the number of transitions from overviews to more intensive reading?

- If no transition were necessary (i.e. if there were a stable reading surface like a Tablet PC), would people behave in the same way?

- When do people prefer to work from metadata to perform this kind of task?

To answer these questions and to delve deeper into understanding the approach followed by users during triage activity, we envisioned a scenario with users reading on a tetherless pen-based tablet computer and consulting a secondary peripheral display (possibly a projected image) to see a metadata-based document overview. Figure 1 shows the envisioned configuration with a person reading on a tablet computer with a peripheral display for organizing documents.

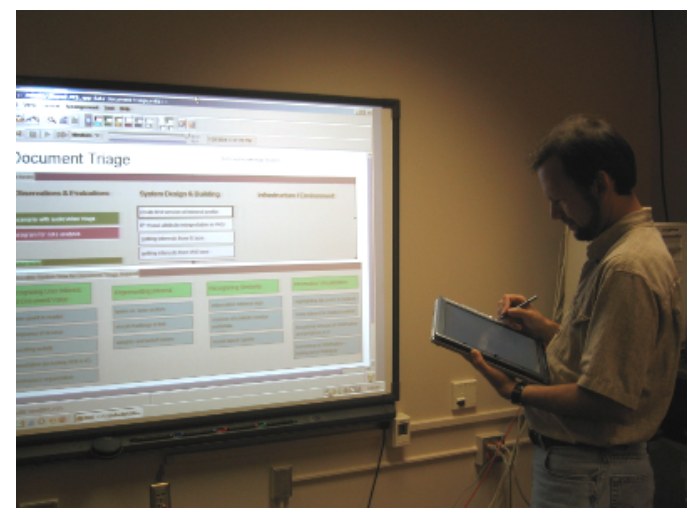

Fig. 1. Envisioned setting includes a reading interface on tetherless tablet computer and an organizing interface on a peripheral display

To investigate this document triage configuration, tablet users need to interact with materials on the projected display, since triage ultimately demands that some judgment be made about the documents' relative merit. Current tablet operating systems are limited in that the pen cannot provide direct input to a second display connected to the same computer. Techniques such as Pick-and-Drop [9] and hyperdragging [10] have been proposed to overcome this limitation, but they require considerable additions/modifications in both hardware and software. We decided to focus on hardware configurations that are common in labs or offices. Two factors that are likely to alter the triage task are the number of displays and the assignment of specific user activity to displays.

\subsection{Multiple Displays}

Research indicates that multiple monitor systems can help users be more productive [2]. Hutchings and colleagues found users with multi-monitors switch windows less frequently than users with single displays [4]. Grudin looks into dual-monitor 
situations [3] and observes that users do not use the additional monitor as "additional space", i.e. they rarely straddle a window across two monitors. Another observation in Grudin's work is that users distribute tasks among monitors; typically using one monitor for the "main" or "primary" task and using the other monitor for "secondary" or "other" tasks. All this suggests that having multiple displays should be effective for triage activity, wherein the user can use one of the displays for reading and the other display to see the document overview. Even though much work has gone into evaluating the efficacy of multiple displays for primary and peripheral tasks, few evaluations directly compare different display configurations for multi-application tasks requiring frequent shifts between applications.

User practice is likely to be impacted by the size of displays used, the distance between the primary and the secondary displays, and the resolution of the displays. The most common configuration is having two displays placed side by side, as occurs with most dual display desktop computers or when an external desktop monitor is attached to a notebook computer. A less common configuration is to have one display near the user and another larger display a few feet away. This occurs when a laptop computer is brought into a meeting room with a plasma or projected display. The first scenario means that the user will not have to readjust her focus when shifting from one display to another. In the second scenario, the user has to constantly refocus (because of the differing distance from the user to the two displays).

\subsection{System/User -Assigned Roles}

Subjects in the study referred to earlier [12] used a single monitor, requiring them to use the same display for both reading and document overview. Given the limited screen real estate of a desktop display, users most often used the entire screen for either reading or document overview. In other words, window overlapping was preferred over tiling of windows. With the introduction of dual/multiple displays for the task, there is the question of how the two displays should be controlled and how tasks should be allocated to the different displays. Should the user be able to move across the two screens seamlessly, i.e. is the "extended desktop" metaphor the most effective? For the second "multiple display" scenario mentioned above, would users prefer to read on the primary display and use the farther (bigger) display for document overview? Should fixed roles be assigned to the two displays, i.e. should there be a mechanism whereby the user is restricted to read on a particular display and manipulate the document overview on the other?

The following study includes three configurations with different combinations of positioning dual displays and user choice of activities on them.

\section{Study Design}

This study investigates the impact of display configuration on document triage practice. The study took place in the Center for the Study of Digital Libraries at Texas A\&M University. Twenty four subjects were recruited via flyers and mass email. 96\% of subjects were from the Computer Science Department and $87 \%$ were graduate students. From discussions with study participants it was determined that some of 
them had previous experience in working with multiple displays. Additionally, $96 \%$ were regular computer users for five or more years. Pre-task interviews indicated that while $80 \%$ of the subjects use computers to read informational web pages (i.e. short newspaper articles, reviews, magazines etc.), only $38 \%$ read long documents (i.e. a 20 page paper) on the screen.

The subjects were placed in the role of a research librarian that had to select and organize documents for a high school teacher preparing a class on ethnomathematics (the study of a group's culturally-specific mathematical practices as they go about their everyday activities). Subjects started with twenty documents returned from the National Science Digital Library (NSDL) and twenty documents returned from Google placed in lists in the Visual Knowledge Builder (VKB), a spatial hypertext system [11]. VKB allows users to organize information objects (links to websites in this study) in a hierarchy of two-dimensional workspaces. None of the subjects had prior experience with VKB and all were given a brief training session to explain the features considered relevant for this task. The forty links to the NSDL and Google documents as well as their arrangement on the VKB space as objects were the same for all subjects. The documents varied in their level of difficulty, relatedness to the topic and volume of information. Subjects were told to take as much time as necessary to complete the task.

This is the same task, setting, and topic as the study reported in [12]. Some of the documents changed between the two studies as the documents on the Web changed and our caches of these documents did not include all the document subcomponents (e.g. inline images, etc.) These changes were in the content of individual documents and unlikely to influence overall triage practice.

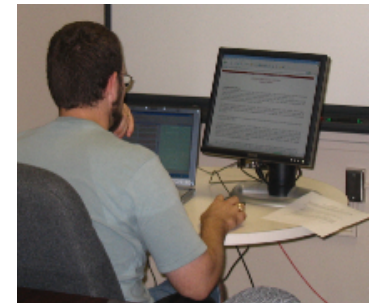

Fig. 2. Group 1 used laptop and tabletop LCD display

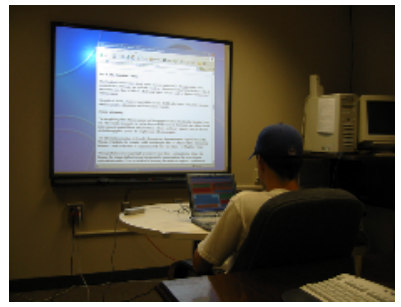

Fig. 3. Group 2 used laptop and projected display

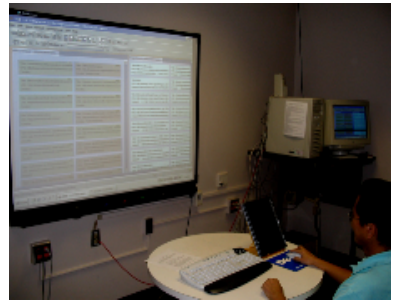

Fig. 4. Group 3 used tablet and projected display

Subjects were randomly divided among three display configurations (table 2). In two of the configurations, subjects had a laptop as a focal display with an extra screen forming an extended desktop. The extra screen was a 17" LCD tabletop monitor placed next to the main display (figure 2) for the first group while the extra screen was a large projected display behind the laptop (figure 3 ) for group 2. In the third configuration, the focal display was a tablet PC while the large projected display was used as the extra screen (figure 4). The subjects of the first two configurations were able to control both screens via keyboard and mouse. They were also free to choose which displays would be for reading and which would be for the document overview. In the third configuration, each display had its own input and control devices (a pen 
for the tablet PC, and keyboard and mouse for the extra screen). Additionally, the role of each display was predetermined in the third configuration. Hence, subjects had to use the tablet PC for reading documents while the large projected display acted as a working space for organizing the links.

Table 2. Characteristics of three multiple display configurations in the study

\begin{tabular}{|c|c|c|}
\hline Display Configuration & Input Devices & Assignment of Activity \\
\hline $\begin{array}{c}\text { Configuration 1: Laptop } \\
\text { and tabletop LCD display. }\end{array}$ & $\begin{array}{c}\text { Extended desktop controlled } \\
\text { via keyboard and mouse. }\end{array}$ & $\begin{array}{c}\text { User controls which } \\
\text { windows are on which } \\
\text { display. }\end{array}$ \\
\hline $\begin{array}{c}\text { Configuration 2: Laptop } \\
\text { and projected display. }\end{array}$ & $\begin{array}{c}\text { Extended desktop controlled } \\
\text { via keyboard and mouse. }\end{array}$ & $\begin{array}{c}\text { User controls which } \\
\text { windows are on which } \\
\text { display. }\end{array}$ \\
\hline $\begin{array}{c}\text { Configuration 3: Tablet } \\
\text { computer and projected } \\
\text { display. }\end{array}$ & $\begin{array}{c}\text { Projected display controlled } \\
\text { via keyboard and mouse, } \\
\text { tablet controlled via pen. }\end{array}$ & $\begin{array}{c}\text { Software assigns document } \\
\text { overview to projected } \\
\text { display and IE to tablet. }\end{array}$ \\
\hline
\end{tabular}

Only basic functionality from IE and VKB was necessary to examine and organize the forty documents. The subjects had to double-click on the VKB objects in order to open the related links and then, based on the content of the documents, organize the links into visual structures for the high school teacher. Participants had to determine their own criteria for including and excluding links and for creating the structures provided to the teacher. They were also free to add text or annotation to their structures in order to make them more understandable and complete.

A variety of data was collected during the study. Screen capture software was used to record on-screen activity on both displays. Video recordings from behind the subjects were recorded to determine the subjects' focus of attention. Additionally, user actions in VKB and IE were logged and provide event times, URLs and Internet Explorer window identifiers. After the task, subjects responded to questionnaires concerning their experience, asked to identify five high-value and five low-value documents from the task, and then took part in an interview based on their activity and answers to the questionnaire.

\section{Results}

The data below includes the three settings from this study as well as the single-display setting from our prior study when it is directly comparable to the current results. The next section includes results concerning the number of transitions between and time spent in the two applications based on log files and video analysis. After this are results examining the relationship between subjects' assessment of document value and their time and activity spent on documents. Finally, results related to subjects' overall perception of the task, the context, and their performance are presented. 


\subsection{Time Spent on Task and Number of Transitions}

Determining the impact of display configuration on the number of transitions and time spent in VKB and IE required combining the results from the log files and videos. Log files provided data on the user interactions such as opening documents for reading, scrolling and mouse clicks in IE, and changes to the organization of links in VKB. To determine changes in subjects' focus of attention, it was also necessary to analyze the videotapes to identify when subjects switched attention between VKB and IE without causing events logged by either IE or VKB. This was primarily determined by changes in head and body position. One limitation is that it was sometimes difficult to recognize the head movement of subjects according to their individual styles in reading and organizing. This was particularly true in the laptop and LCD screen case because head movement was relatively subtle compared to the projected display configurations. We expect this increases the margin of error about the number of transitions in the laptop and LCD screen configuration.

Table 3. Time spent and number of transitions in four different configurations

\begin{tabular}{|l|r|r|r|r|}
\hline & Prior Study & \multicolumn{3}{|c|}{ Current Study } \\
\hline Configuration & Desktop PC & $\begin{array}{l}\text { Laptop \& } \\
\text { LCD } \\
\text { screen }\end{array}$ & $\begin{array}{l}\text { Laptop \& } \\
\text { projected } \\
\text { display }\end{array}$ & $\begin{array}{l}\text { Tablet PC \& } \\
\text { projected } \\
\text { display }\end{array}$ \\
\hline \# of displays & 1 & 2 & 2 & 2 \\
\hline Avg. total time (sec) & 3,309 & 3,554 & 3,642 & 4,234 \\
\hline Avg. time in VKB (sec) & 2,359 & 2,453 & 2,627 & 3,005 \\
\hline Avg. time in IE (sec) & 950 & 1,102 & 1,015 & 1,229 \\
\hline Avg. \# of transitions & 97 & 193 & 168 & 205 \\
\hline $\begin{array}{l}\text { Avg. \# of documents } \\
\text { visited }\end{array}$ & $\begin{array}{r}\text { Data not } \\
\text { available }\end{array}$ & 34.38 & 30.88 & 31.88 \\
\hline
\end{tabular}

Time on Task. Average total time in the dual display configurations is 3,810 seconds, which is $15 \%$ higher than that in the single display configuration of 3,723 seconds. Normality tests indicated that the distribution of total time spent on task was normal but the time spent in IE was not. Thus, different statistical tests were necessary to assess significance. The difference in total time is not significant by t-test $(p=0.365)$. Average total time of Tablet PC and projected display is the highest among the four configurations, but the difference is not significant by ANOVA test $(p=0.575)$. Average time in IE in the dual display configurations is 1,115 seconds, which is $17 \%$ higher than that in the single display configuration of 950 seconds. However, this difference is not significant by Mann-Whitney test at significance level 0.05. Average time in IE of Tablet PC and projected display is the highest one among four configurations, but the difference is not significant by Kruskal-Wallis test at significance level 0.05 .

In addition, we have examined the percentage of time in IE over total time: the percentage is consistent over the four configurations, around $30 \%$. 
Number of Transitions. On average, there were 189 transitions in the dual display configurations, compared to 97 on average in the single display configuration (Figure 5a). The difference is significant by t-test $(\mathrm{p}=0.002)$.

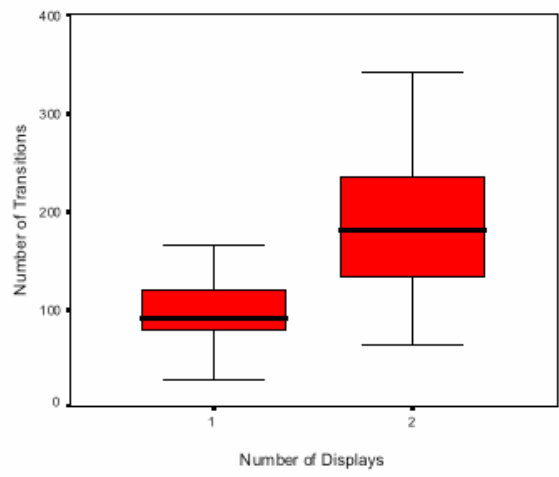

(a)

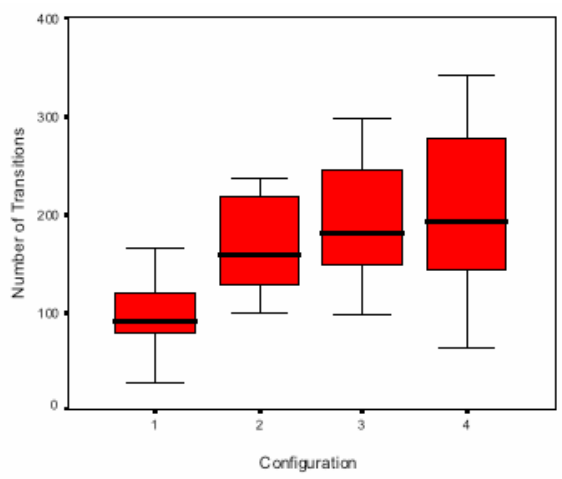

(b)

Fig. 5. Number of transitions for (a) different numbers of displays and (b) four different display configurations. The configurations in (b) are 1: Single Display (prior study), 2: Laptop and extra screen, 3: Laptop and projected display, 4: Tablet PC and projected display.

ANOVA test shows that average number of transitions among four configurations is not all equal ( $\mathrm{p}=0.013)$. Post hoc tests (Turkey and LSD) show the single display configuration is significantly different from Laptop and Extra screen and Tablet PC and projected display, but not significantly different from Laptop and projected display at significance level 0.05 . The average number of transitions for the Tablet PC and projected display is the highest among the three dual display configurations, but the post hoc tests show that the difference is not significant at significance level 0.05 .

\subsection{User Behavior in Reading and Evaluation of Documents}

Each subject was given 20 documents from NSDL and 20 documents from Google, but did not read all the documents: they did not open $19 \%$ of the documents in IE.

Document Value and User Behavior. After subjects finished their tasks, they were asked to select the five least useful documents and the five most useful documents. To estimate overall document value, these results were aggregated. Each time a document was listed among the most useful documents, it was received 2 points. When it was listed among the least useful documents, it received 0 points. If not contained on either list, it received 1 point. We summed up document scores of each document and sorted documents by the document score.

Determining the time spent interacting with a particular document was complicated by the fact that the log files did not recognize transitions from reading in IE to looking at the document organization in VKB when subjects did not generate any events. This resulted in a significant overestimation of the time spent in IE and on reading 
documents. Video analysis indicated that this problem was primarily at the beginning and end of time spent in IE. Therefore, we built an algorithm to estimate the time spent in IE and the time spent in VKB in between logged events indicating a transition based on the results of the prior study. This estimation better matched the video analysis results in terms of total time, total time in IE, number of transitions, and the proportion of time in IE over total time.

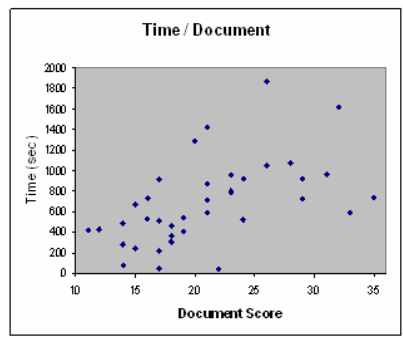

(a)

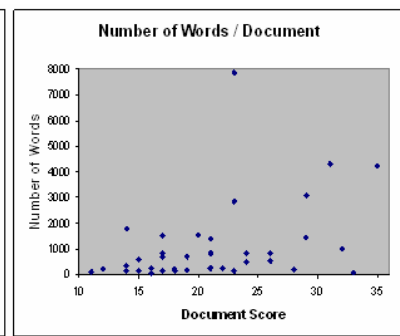

(b)

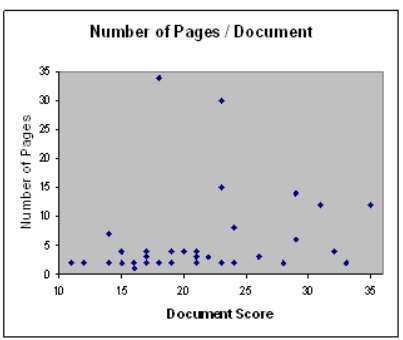

(c)

Fig. 6. Graphs showing document score and (a) time on documents, (b) the number of words in a document, and (c) the number of pages in a document

Figure 6a graphs the sum of time spent on each document across the 24 subjects and its accumulated document score. Correlation analysis shows that time spent is positively correlated to document score (Pearson coefficient $=0.532$ and $p=0.001$ ). As for document style, we examined the number of HTML links in a document, the number of images in a document, file size, the number of words in a document and the number of pages of a document, where the number of pages is the number of $1024 \times 768$ screens needed to display the document. Correlation analysis shows that the number of words and number of pages are both positively correlated to document score (Pearson coefficient $=0.397$ and $0.351, \mathrm{p}=0.015$ and 0.033 ). These graphs are shown in Figure $6 \mathrm{~b}$ and $6 \mathrm{c}$. Two subjects mentioned the length of documents as a characteristic when choosing the five most useful documents. We have not found any significant correlation between document score and other document style attributes.

We examined the correlation between document score and five user events: scrolls, mouse clicks, text selections, the number of times subjects followed embedded links on a document, and the number of visits on a document. Correlation analysis suggests that scroll event is highly correlated to document score as shown in Figure $7 \mathrm{a}$ (Pearson coefficient $=0.632, \mathrm{p}<0.0001$ ). The number of visits on a document, mouse clicks, and text selections are positively correlated to document score (Pearson coefficient $=0.480,0.354$, and 0.331, $\mathrm{p}=0.003,0.034$, and 0.049). However, the number of times subjects followed embedded links on a document is negatively correlated to its document score (Pearson coefficient=-0.334, $\mathrm{p}=0.040$ ).

A variety of user behavior (time spent on documents, scrolls, mouse clicks, text selections, the number of times subjects followed embedded links on a document, and the number of visits on a document) and document attributes (the number of words and the number of pages) are correlated to the perceived value of documents. 


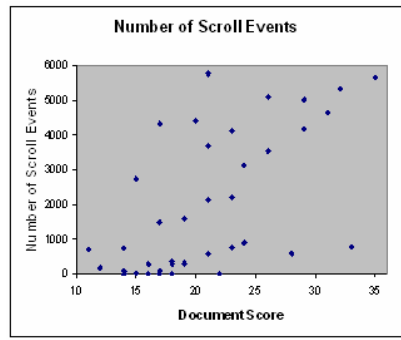

(a)

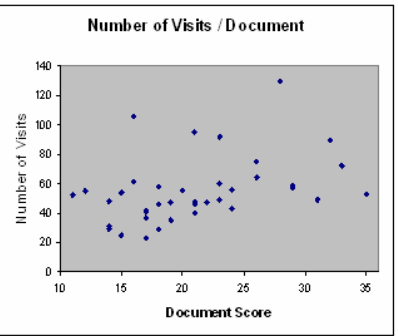

(b)

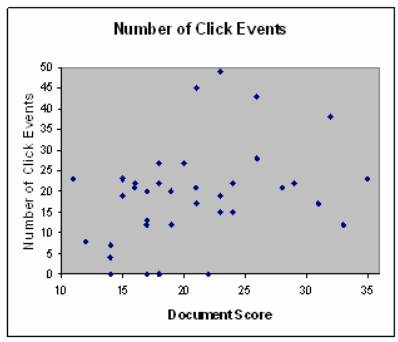

(c)

Fig. 7. Correlation between document score and (a) the number of scroll events, (b) the number of visits on a document, and (c) the number of click events

\subsection{Questionnaire Results}

Table 4 presents the results from five-point Likert scale questions regarding subjects' experience with the hardware and software where 1 was strongly disagree and 5 was strongly agree.

Table 4. User assessment of the display configurations: 1: Single Display (prior study), 2: Laptop and extra screen, 3: Laptop and projected display, 4: Tablet PC and projected display

\begin{tabular}{|l|c|c|c|c|}
\hline $\begin{array}{l}\text { Q1: I feel comfortable reading documents on a } \\
\text { computer. }\end{array}$ & N/A & 4.1 & 3.3 & 3.3 \\
\hline $\begin{array}{l}\text { Q2: It will be easy for someone else to understand the } \\
\text { way I organized the documents. }\end{array}$ & 4.1 & 3.9 & 3.5 & 3.9 \\
\hline $\begin{array}{l}\text { Q3: It will be easy to go back later and understand the } \\
\text { rationale behind my organization. }\end{array}$ & 4.3 & 4.4 & 3.9 & 4.0 \\
\hline Q4: I enjoyed doing this task. & 3.8 & 3.9 & 4.1 & 3.5 \\
\hline $\begin{array}{l}\text { Q5: A tablet PC/laptop is effective in reading } \\
\text { documents. }\end{array}$ & N/A & 3.0 & 3.0 & 3.1 \\
\hline $\begin{array}{l}\text { Q6: I was able to operate the tablet PC/laptop as I } \\
\text { wanted. }\end{array}$ & N/A & 3.4 & 4.3 & 3.3 \\
\hline Q7: It was easy doing the task with two displays. & N/A & 4.1 & 4.4 & 4.0 \\
\hline $\begin{array}{l}\text { Q8: Simultaneously viewing VKB and IE windows on } \\
\text { different displays was helpful in reading documents. }\end{array}$ & N/A & 4.1 & 4.4 & 4.4 \\
\hline $\begin{array}{l}\text { Q9: Simultaneously viewing VKB and IE windows on } \\
\text { different displays was helpful in organizing links. }\end{array}$ & N/A & 4.1 & 4.1 & 4.1 \\
\hline
\end{tabular}

Question 1 indicates that among the three dual display configurations, the subjects in Laptop and Extra Screen felt more comfortable over the subjects in other two configurations. This could be the result of the display characteristics of projected displays (e.g. decreased contrast and image quality) and the different focal distance for the user. The Tablet PC and projected display users had relatively low assessment of their task enjoyment (Question 4) and ability to operate the computers as they wanted (Question 6). We suspect that the pen-based interface in this configuration 
contributed to these results as three subjects mentioned difficulty manipulating the documents they were reading with the pen-based interface. Questions 7 to 9 indicate that subjects in all the three dual display configurations felt that the dual display environments were helpful for their given tasks.

\subsection{Interview Results}

Subjects were interviewed concerning their organizational strategies, methods for evaluating documents, and experiences with their dual display configuration.

With regards to organizational strategies, 13 subjects employed high-level categorization strategies such as dividing resources into books, papers, link collections, and by source of information. These high-level strategies limited the amount of reading required. 17 subjects employed categorization schemes based on an assessment of the content of documents such as their relevance, and whether they were introductory or professional. Notice that six subjects combined both high-level categorizations of documents and an assessment of their contents.

When asked about the evaluation of document value, 16 subjects mentioned characteristics of the content of documents: document providing a good introduction (7), documents giving information directly (7), and the amount of information (2). In contrast, only 3 subjects mentioned document structure or format (hierarchy, embedded links and document file format).

When asked about the role of document metadata provided in $\mathrm{VKB}$, such as page title, page URL and summary, 14 subjects reported that they read the page title before reading documents. Among those 14 subjects, 4 paid attentions page URL as well, and 3 paid attentions to page URL and summary as well. 13 subjects examined the metadata after reading documents, while 4 subjects did not. However, the primary object of revisiting the metadata was to identify documents that they previously read. When we asked subjects what extra information would be useful in the document overview for a better understanding of the web site content, 9 subjects said keywords for the Web pages, while 7 subjects requested thumbnail images of the documents.

When asked about display configurations, 8 subjects preferred the two displays next to each another, while 7 subjects preferred a screen able to display more content. In addition, 5 subjects answered that they preferred one display in front and another display in the background.

\section{Discussion}

The data gathered from the study lends itself to observations concerning the impact of display configuration on document triage practice and the relationship between user activity and perceived document value.

Our central question on the impact of multiple displays on transitions between applications is partially answered by the data in Table 3 . The number of transitions in the single display case is almost half the number of transitions in the dual display case (averaging over the three dual display cases). This would seem surprising at first, given that transitions were sources of interruption as the users had to deal with window management for every transition in the single display case; but the data 
indicates that users do not see transitioning between the two displays as bothersome, as the effort required in shifting attention between the two displays is negligible. With two displays, users chose to switch between the document overview and the document more often, perhaps working less from metadata. This and the positive responses received about the dual display configurations for questions Q7, Q8 and Q9 in the questionnaire suggest that having two displays is a more "natural" setting for document triage.

Comparing the average number of transitions between the three dual display configurations, it is seen that the number of transitions in the Laptop and Projected Display configuration (LPD) is the least, the number of transitions in the Tablet PC and Projected Display configuration (TPD) is the highest, and the number in Laptop and Extra Screen configuration (LES) was nearly as high as the TPD configuration. Even though the differences are not statistically significant, it is worthwhile to look at the factors influencing them. One difference between configurations LES and LPD is the focal distance between the primary and secondary displays. Since in LES both screens are at the same focal distance, being side by side, the effort required in shifting focus between the screens is lower compared to the LPD case. The qualitative data from the post-task interviews supports this hypothesis. When asked which display configuration they would prefer, many people ( 9 of the 17 asked, including 6 from the two subjects who had used the projected display configurations) answered "two screens, one next to the other". Some subjects also complained about the size of the projected display saying that it was annoying to look back and forth.

The difference in the number of transitions between configurations LPD and TPD may have been influenced by two characteristics of the configurations. The first difference is that there are two separate input devices in the TPD case (keyboard and mouse controlling the projected display, and the pen controlling the Tablet PC), whereas in the LPD case, the same keyboard and mouse act upon both displays. We expected that the need to switch between the different input devices might reduce the number of transitions in the TPD case. Clearly, this did not deter the subject's from transitioning between the two displays. The second difference between the LPD and TPD configurations is whether users could choose which display to use each activity. In the TPD configuration, the roles of the two displays are fixed, so when the user "opens" a document in the document overview on the projected display it is automatically presented on the tablet computer. This reduced the user's task of window management. Further study is necessary to determine the relative influence of the reduced overhead of window management and the increased effort of switching input devices in the TPD configuration.

The number of documents visited by the subjects in the LES configuration is slightly more than it is for the other two configurations. While not statistically significant, this may further indicate that having two displays at the same focal distance is better suited for a thorough and efficient triage activity.

One unexpected finding was that subjects with a single display took less time to finish the task, although not significantly, than subjects with multiple displays (see table 3). The fastest and the slowest group from those who used multiple screen settings (LES and TPD respectively) performed 7.4\% and 28\% slower than the group with the single display. A possible explanation is that subjects took advantage of the multiple screen environment and spent more time skimming and reading documents 
before organizing links. Without the window management disruption, subjects were more willing to switch views and make an effort at evaluating actual document content before judging the document's value.

But willingness to read document content was probably not the only determining factor in the added time for the TPD configuration. Many subjects had complaints and difficulties using the pen as a control device of the tablet PC (scrolling was reported as one of the most painful and time consuming tasks). Comments during post-task interviews as well as the responses to the question about ease of operating the hardware (Q6, table 4), and the question about enjoyment of task (Q4, table 4), indicate subjects preferred the mouse and keyboard while reading documents in IE.

Subjects spent a widely variable amount of time reading/scanning/skimming the content of individual documents. While this was expected, we were unclear how the extra attention a document receives is related to perceived document value. Would subjects quickly assess the best and worst documents and spend more time on those in the "middle of the pack", or would they become more critical of documents as they looked at them longer? The data analysis shows that the time spent in a document is positively related to the user's interest in the document. It is already known that reading time for in-depth reading is an indicator of user interest in a document ([1], [8]). Our data strengthens this previous finding; i.e. time spent in a document is an indicator of interest even when the user is skimming/scanning for the purpose of document triage.

There were also relations between perceived document value and certain user events, as was previously seen by Kelly and Belkin [6]. The number of scroll events to a particular document indicated a higher perceived document value. Also, we find the number of pages in a document and the number of words in a document to be related to user interest, as well. Document length seems to have strongly influenced these findings. In addition to these two objective measurements, document length likely impacted the number of scroll events of subjects.

In the interviews that followed the task, subjects were asked as to how they evaluated the usefulness/scope of documents they skimmed over. From the user feedback, page layout and content of the page play a vital role. Most users considered documents with a lot of text as authoritative. But opinion was divided on the usefulness of such documents based on subjects' interpretation of their task. Some users felt that as they were looking for introductory information on ethnomathematics for the high school teacher and determined that documents with a lot of detail were unnecessary. Others felt that the long and detailed documents provided the most information and thus were useful. Many of the users looked at the metadata information provided in VKB (document title and URL) before and after visiting Web pages. This suggests that they had expectations of what they would find at websites such as ethnomath.org, pages in the .edu domain, amazon.com links, and wordspy.com pages.

Pages of pointers to other documents were not perceived as valuable by subjects, as indicated by the number of followed links being negatively correlated to perceived document value. This was confirmed by subjects' comments indicating websites which simply referred to other sites, or which contained a lot of links without actually containing much information, were not useful. 


\section{Conclusions}

Document triage is the practice of rapidly locating, skimming, selecting, and organizing documents for later use. The combination of the rapid nature of document assessment and the use of separate document overview and reading applications creates a large number of window transitions. By comparing document triage practice under multiple display configurations, it was determined that subjects transitioned between applications more often when using multiple displays than they did when using a single display. Additionally, users evaluated documents more by reading their contents and less often relied solely on metadata. Users spent more time reading and interacting with documents that they valued. This confirms prior research showing such correlations between time spent reading and assessed document value during indepth reading carry over into the triage activity. ${ }^{1}$

\section{References}

1. Chan, P.: A Non-Invasive Learning Approach to Building Web User Profiles. In Workshop on Web Usage Analysis and User Profiling (1999) 7-12.

2. Czerwinski, M., Smith, G., Regan, T., Meyers, B., Robertson, G., Starkweather, G.: Toward characterizing the productivity benefits of very large displays. IOS Press, Proceedings of INTERACT (2003) 9-16.

3. Grudin, J.: Partitioning Digital Worlds: Focal and Peripheral Awareness in Multiple Monitor Use. Proceedings of CHI (2001) 458-465.

4. Hutchings, D.R., Smith, G., Meyers, B., Czerwinski, M., Robertson, G.: Display space usage and window management operation comparisons between single monitor and multiple monitor users. ACM Press, Proceedings of AVI (2004).

5. Joyce, M.: The lingering errantness of place. A talk given at the ACRL/LITA Joint Presidents Program, American Library Association, 114th Annual Conference, (1995).

6. Kelly, D., Belkin, N.J. Display time as implicit feedback: Understanding task effects. In Proceedings of SIGIR '04, Sheffield, UK, (2004) 377-384.

7. Marshall, C.C., Shipman, F.: Spatial Hypertext and the Practice of Information Triage. In Proceedings of ACM Hypertext (1997) 124-133.

8. Morita, M., Shinoda, Y.: Information filtering based on user behavior analysis and best match text retrieval. Proceedings of ACM SIGIR Conference. (1994) 272-281.

9. Rekimoto, J.: Pick-and-Drop: A Direct Manipulation Interface for Multiple Computer Environments. Proceedings of UIST (1997) 31-39.

10. Rekimoto, J., Saitoh, M.: Augmented Surfaces: A Spatially Continuous Workspace for Hybrid Computing Environments. Proceedings of CHI (1999).

11. Shipman, F., Hsieh, H., Airhart, R., Maloor, P., Moore, J.M.: The Visual Knowledge Builder: A Second Generation Spatial Hypertext. Proc. of Hypertext (2001) 113-122.

12. Shipman, F., Hsieh, H., Moore, J.M., Zacchi, A.: Supporting Personal Collections across Digital Libraries in Spatial Hypertext. Proceedings of JCDL (2004) 358-367.

\footnotetext{
1 This work was supported in part by National Science Foundation grant DUE 02-26321.
} 